\title{
Is Conization a Protective Surgical Maneuver in Early Cervical Cancer?
}

\author{
Luis Chiva, MD, PhD, and Enrique Chacon, MD \\ Department of Obstetrics and Gynecology, Clínica Universidad de Navarra, Madrid, Spain
}

In an exciting study published in this issue of Annals of Surgical Oncology, Bizarri et al. present a notable working hypothesis, i.e., that cervical conization can have a protective effect that can diminish relapse rates after radical hysterectomy. ${ }^{1}$ The study included 336 patients from various centers across Italy and the UK, collected over 15 years. Using a statistical propensity matching score methodology, it matches one-to-one patients undergoing and not undergoing conization. After comparing the two groups for risk variables, the authors find that patients after conization suffer fewer relapses, have less adjuvant treatment, and do not present more complications due to this previous surgery. This study suffers from the limitations of a retrospective study over a long period without strict inclusion and exclusion criteria; however, the attempted statistical propensity matching score methodology makes it valid hypothesis-generating research.

For decades, we have used cervical conization with a diagnostic purpose, trying to rule out invasion and understand tumor characteristics to plan the extent of surgical treatment. The most frequent indication for conization is reasonable doubt over which therapeutic option is most appropriate.

At some centers, this is systematically carried out in all invasive tumors to discover tumor characteristics and offer adequate treatment. Nevertheless, this is, generally speaking, a rare approach among experts.

(C) Society of Surgical Oncology 2021

First Received: 26 January 2021

Accepted: 28 January 2021;

Published Online: 17 March 2021

L. Chiva, MD, $\mathrm{PhD}$

e-mail: 1chiva@unav.es
With the publication of the study on the LACC trial, it is evident that early cervical cancer treatment with minimally invasive surgery offered worse results in terms of relapse and survival. ${ }^{2}$ The LACC trial prompted significant debate, with the intention of defining the reasons for these findings and potential protective maneuvers to improve these results; For instance, conization was considered, among others, to be an exclusion criterion in the SUCCOR study. ${ }^{3}$ We subsequently came to suspect that it could introduce a relevant bias; however, the LACC trial included patients after conization without considering it to be a confounding variable.

Bizarri et al.'s results coincide and support our research presentation at the recent "State of the Art 2020" ESGO meeting that described similar results in a large European population of 1152 patients undergoing radical hysterectomy during 2013 and 2014. Our findings showed firstly that previous cone biopsy before radical hysterectomy is the most predictive independent variable after meticulously matching the groups. ${ }^{4}$ Secondly, our SUCCOR Cone study demonstrated that patients undergoing conization obtain a clear benefit in terms of disease-free survival (DFS) and overall survival $(\mathrm{OS})^{5}$. This advantage was significant in patients after minimally invasive surgery, who achieved the same relapse and survival results as those who underwent open surgery, again highlighting the protective impact of conization.

Without a doubt, conization can reduce the risk of contact between the tumor and abdominal cavity during surgery, a particularly important maneuver when performing minimally invasive surgery. To the best of the authors' knowledge, conization has never been considered from this perspective before. 
Perhaps the time has come to prospectively evaluate its protective effect by adding or abandoning other suggested maneuvers that could influence the relapse rate, such as abandoning the uterine manipulator and protective closure of the vagina before colpotomy. ${ }^{3}$

At this moment, we can look back in awe at the great pioneers in radical cervical cancer surgery, Ernst Wertheim and Frederick Shauta, who dealt with giant tumors with only their hands. At the beginning of the twentieth century, they worked by systematically carrying out resection of the visible tumor before performing their radical surgery, thus avoiding dissemination. Therefore, we congratulate Bizarri et al. for their interesting hypothesis, which must be validated. In the meantime, we must contemplate that conization may have a therapeutic effect beyond what is currently known in treatment of early cervical cancer.

DISCLOSURE Luis Chiva de Agustin-Grants from Astra Zeneka, Roche and GSK

\section{REFERENCES}

1. Bizzarri N, Pedone Anchora L, Kucukmetin A, et al. Protective role of conization before radical hysterectomy in early-stage cervical cancer: a propensity-score matching study. Ann Surg Oncol. 2021. https://doi.org/10.1245/s10434-021-09695-4.

2. Ramirez PT, Frumovitz M, Pareja R, et al. Minimally invasive versus abdominal radical hysterectomy for cervical cancer. $N$ Engl J Med. 2018;379:1895-904.

3. Chiva L, Zanagnolo V, Querleu D, On behalf of the SUCCOR study Group, et al. SUCCOR study: an international European cohort observational study comparing minimally invasive surgery versus open abdominal radical hysterectomy in patients with stage IB1 cervical cancer. Int J Gynecol Cancer. 2020;30:1269-77.

4. Manzour N, Chacon E, Martín-Calvo N, et al. Design and validation of a recurrence risk predicting score in early stage cervical cancer after radical hysterectomy. Int J Gynecol Cancer. 2020;30:A8.

5. Chacón E, Manzour N, Mínguez JÁ, et al. Succor cone: is it cervical conization a protective maneuver. Int J Gynecol Cancer. 2020;30:A7-8.

Publisher's Note Springer Nature remains neutral with regard to jurisdictional claims in published maps and institutional affiliations. 